\title{
Screening Patients with Esophageal Cancer to Determine Eligibility for Adjuvant Treatment Trials
}

\author{
TAHER ABU HEJLEH ${ }^{1,2}$, LAITH ABUSHAHIN ${ }^{1}$, JOHN KEECH ${ }^{3}$, BRYAN G. ALLEN ${ }^{4}$, \\ KALPAJ R. PAREKH ${ }^{3}$, MUHAMMAD FURQAN ${ }^{1}$, MARK IANNETTONI ${ }^{5}$, \\ GIDEON K.D. ZAMBA ${ }^{6,7}$, SARAH L. MOTT ${ }^{7}$ and GERALD CLAMON ${ }^{1}$ \\ ${ }^{1}$ Division of Hematology/Oncology, Department of Internal Medicine, \\ ${ }^{3}$ Division of Thoracic and Cardiovascular Surgery, Department of Surgery, ${ }^{4}$ Department of Radiation Oncology, \\ ${ }^{6}$ Department of Biostatistics and ${ }^{7}$ Holden Comprehensive Cancer Center, \\ University of Iowa Hospitals and Clinics, Iowa City, IA, U.S.A.; \\ ${ }^{2}$ Division of Hematology, Oncology, and Blood \& Marrow Transplantation, \\ Department of Internal Medicine, King Hussein Cancer Center, Amman, Jordan; \\ ${ }^{5}$ Department of Cardiovascular Sciences, Brody School of Medicine at East Carolina University, Greenville, NC, U.S.A.
}

\begin{abstract}
Background: The tolerability of adjuvant chemotherapy in esophageal cancer is unclear. Patients and Methods: This was a phase II trial of adjuvant paclitaxel in patients with esophageal cancer after trimodality treatment. Patients with residual viable tumor after resection were eligible for study inclusion. Treatment was $80 \mathrm{mg} / \mathrm{m}^{2}$ paclitaxel intravenously on days 1,8 , and 15 every 28 days for total of two cycles. The primary objective was to determine whether $75 \%$ or more of the patients would tolerate $240 \mathrm{mg} / \mathrm{m}^{2}$ or more of paclitaxel, which corresponded to $50 \%$ or more of the total planned dose. Results: Eleven out of the 12 enrolled patients (92\%, 95\% confidence interval $(\mathrm{CI})=62-100 \%$ ) were able to complete at least $50 \%$ of the planned paclitaxel dose. Median progression-free survival was 7 months $(95 \%$ CI $=2-28$ months). Median overall survival was 28 months $(95 \%$ CI=12-36 months). Only one patient experienced a grade 4 adverse event. Conclusion: Screening patients with esophageal cancer after trimodality treatment might improve completion of adjuvant trials.
\end{abstract}

The standard treatment for resectable esophageal cancer is concurrent chemotherapy and radiation therapy followed by esophagectomy, which showed survival benefit over surgery alone (1). Despite this trimodality approach, there is a high

Correspondence to: Gerald Clamon, MD, Department of Internal Medicine, 200 Hawkins Drive, University of Iowa Hospitals and Clinics, Iowa City, IA 52242, U.S.A. Tel: +1 3193561932, Fax: +1 3193538383, e-mail: gerald-clamon@uiowa.edu

Key Words: Esophageal cancer, adjuvant chemotherapy. risk of cancer recurrence and ultimately death. Overall survival at 5 years in the Cross trial for patients who received neoadjuvant chemoradiation then esophagectomy was $47 \%$ (1). Among many clinical and pathological features considered to be related to cancer recurrence, less than complete pathological response to neoadjuvant treatment is viewed as an important feature that correlates with cancer recurrence and poor survival (2). Thus, effective adjuvant treatments in esophageal cancer are desperately needed.

The benefit from adjuvant systemic therapy in patients who undergo resection of esophageal cancer is unclear (3-8). Adjuvant chemotherapy following esophagectomy has been challenging due to the toxicity associated with treatment $(5,6)$. In the MAGIC trial, only $55 \%$ of the randomized patients were able to receive any of the postoperative chemotherapy and only $42 \%$ completed all intended adjuvant therapy (5). In a trial testing sunitinib as adjuvant treatment in esophageal cancer, $41 \%$ of the patients discontinued the treatment prematurelymostly due to toxicity (6). The benefit noticed in adjuvant systemic therapy in esophageal cancer trials has been limited to disease-free survival, without significant improvement in overall survival $(3,7)$. Currently, adjuvant chemotherapy alone is not the standard of care for resected esophageal cancer, but adjuvant chemoradiation is recommended if radiation was not delivered in the neoadjuvant setting.

Among various chemotherapies tested in esophageal cancer, paclitaxel is an active drug as a single agent or in combination regimens. A phase II trial showed that paclitaxel as a single agent had a $31 \%$ overall response rate in esophageal cancer (9). Another phase II trial testing the combination of paclitaxel, cisplatin and 5-fluorouracil in unresectable esophageal cancer showed tumor response in $48 \%$ of cases (10). 
In this trial, the goal was to determine whether screening patients with esophageal cancer for enrollment in an adjuvant paclitaxel clinical trial after esophagectomy, rather than before starting neoadjuvant treatments, may improve the completion rate of adjuvant chemotherapy delivery. Although this approach will result in selection bias due to enrolling 'healthier' patients in the trial, this design might allow for more definitive assessment of the benefit of adjuvant treatment in patients who actually receive the adjuvant treatment. Currently, the benefit from adjuvant treatment in esophageal cancer is unclear due to the shortcomings of poor tolerance and high discontinuation rates.

\section{Patients and Methods}

This was a single arm study conducted at the University of Iowa Hospitals and Clinics to assess the tolerability of paclitaxel as adjuvant chemotherapy post-surgery for patients who had received neoadjuvant chemoradiotherapy. The study had local Institutional Review Boards review and approval (NCI CTRP number: NCI2012-00426). Data management and analysis were conducted onsite and the trial was monitored by the University of Iowa Data and Safety Monitoring Committee.

The main eligibility criteria for enrolling in the trial included a diagnosis of esophageal or gastroesophageal cancer requiring neoadjuvant chemotherapy and radiation therapy followed by esophagectomy, residual viable cancer in the esophagus or nodes in the surgical specimen after neoadjuvant therapy and R0 resection. Eligible patients had to be 18 years or older. Patients were only eligible to enroll if they had recovered sufficiently from surgery to be able to eat and maintain their weight. Patients with a prior malignancy in the previous 5 years except resected non melanomatous skin cancer or in situ cervical carcinoma were excluded. Patients had to have a performance score of 0-2 and were to have completed $80 \%$ of the planned neoadjuvant therapy. Patients were required to have absolute neutrophil count $>1800 / \mathrm{mm}^{3}$, platelets $>100,000 \mathrm{~mm}^{3}$, creatinine $<1.5 \mathrm{mg} / \mathrm{dl}$, total bilirubin $<1.2$ $\mathrm{mg} / \mathrm{dl}$, and aspartate aminotransferase and alanine aminotransferase $<$ twice the upper limit of normal. Patients with postoperative dumping syndrome or uncontrolled infection were excluded. Patients with morbid obesity (body mass index $>40 \mathrm{~kg} / \mathrm{m}^{2}$ ), those who required a colon interposition, who were unable to provide informed consent, who had significant other medical conditions, or who were unable to start adjuvant therapy in 8 weeks \pm 10 days from the time of esophagectomy were excluded.

The neoadjuvant chemotherapy and radiation therapy were not specified for this trial and were intended to be standard of care per the treating physician's choice. Radiation treatment was to be delivered using 6,10 , or $15 \mathrm{MV}$ photons using either $3 \mathrm{D}$ conformal or intensity-modulated radiation therapy depending on optimal dose distribution as determined by the attending radiation oncology physician. Target volume delineation consisted of gross tumor volume (GTV), including clinically or pathologically involved lymph nodes identified by computed tomography (CT) or positron emission tomography (PET) imaging. clinical target volumes (CTV) were generated to cover at-risk nodal regions and submucosal extension of the tumor along the length of the esophagus/proximal stomach. Planning target volumes (PTV) were generated to account for set-up
Table I. Patient and clinical characteristics.

\begin{tabular}{lcc}
\hline Variable & $\mathrm{N}$ & $\%$ \\
\hline Gender & 3 & \\
Female & 9 & 25.0 \\
Male & & 75.0 \\
Smoker & 6 & 50.0 \\
No & 6 & 50.0 \\
Yes & & \\
Clinical stage at diagnosis* & 1 & 9.1 \\
T2N1 & 3 & 27.3 \\
T3N0 & 4 & 36.4 \\
T3N1 & 3 & 27.3 \\
T3N2 & 1 & - \\
Not fully staged & & \\
Postoperative pathologic stage & 1 & 8.3 \\
T1aN1 & 1 & 8.3 \\
T1bN1 & 1 & 8.3 \\
T2N1 & 3 & 25.0 \\
T3N0 & 5 & 41.7 \\
T3N1 & 1 & 8.3 \\
T3N2 & & Range \\
\hline & Median \\
\hline Age, years & 57.5 & $33.0-74.0$ \\
BMI, kg/m ${ }^{2}$ & 29.7 & $18.6-33.7$ \\
\hline
\end{tabular}

BMI: Body mass index. *Based on some combination of endoscopic ultrasound, computed tomography and positron emission tomography.

Table II. Maximum grade 3-5 adjuvant paclitaxel attributable toxicities in 12 assessable patients.

\begin{tabular}{llll}
\hline & \multicolumn{3}{c}{ Grade } \\
\cline { 2 - 4 } Adverse event & 3 & 4 & 5 \\
\hline Lymphocyte count decreased & 4 & 1 & 0 \\
Neutrophil count decreased & 1 & 0 & 0 \\
White blood cell count decreased & 2 & 0 & 0 \\
\hline
\end{tabular}

error and movement. Radiation dose was delivered in $1.8 \mathrm{~Gy}$ fractions, with total dose ranging from $41.4 \mathrm{~Gy}$ in one patient treated at another hospital to $50.4 \mathrm{~Gy}$ in the 11 other patients.

The neoadjuvant chemoradiation in 10 of the patients had been paclitaxel and carboplatin concurrent with radiation. One patient received neoadjuvant cisplatin and fluorouracil infusion concurrent with radiation and another received cisplatin, fluorouracil and oxaliplatin concurrent with radiation. The planned adjuvant therapy on study consisted of administration of $80 \mathrm{mg} / \mathrm{m}^{2}$ paclitaxel intravenously on days $1,8,15$ every 28 days for a total of two cycles. Doses were calculated using actual body surface area. The total cumulative planned dose for the 6 weeks of therapy was $480 \mathrm{mg} / \mathrm{m}^{2}$. 


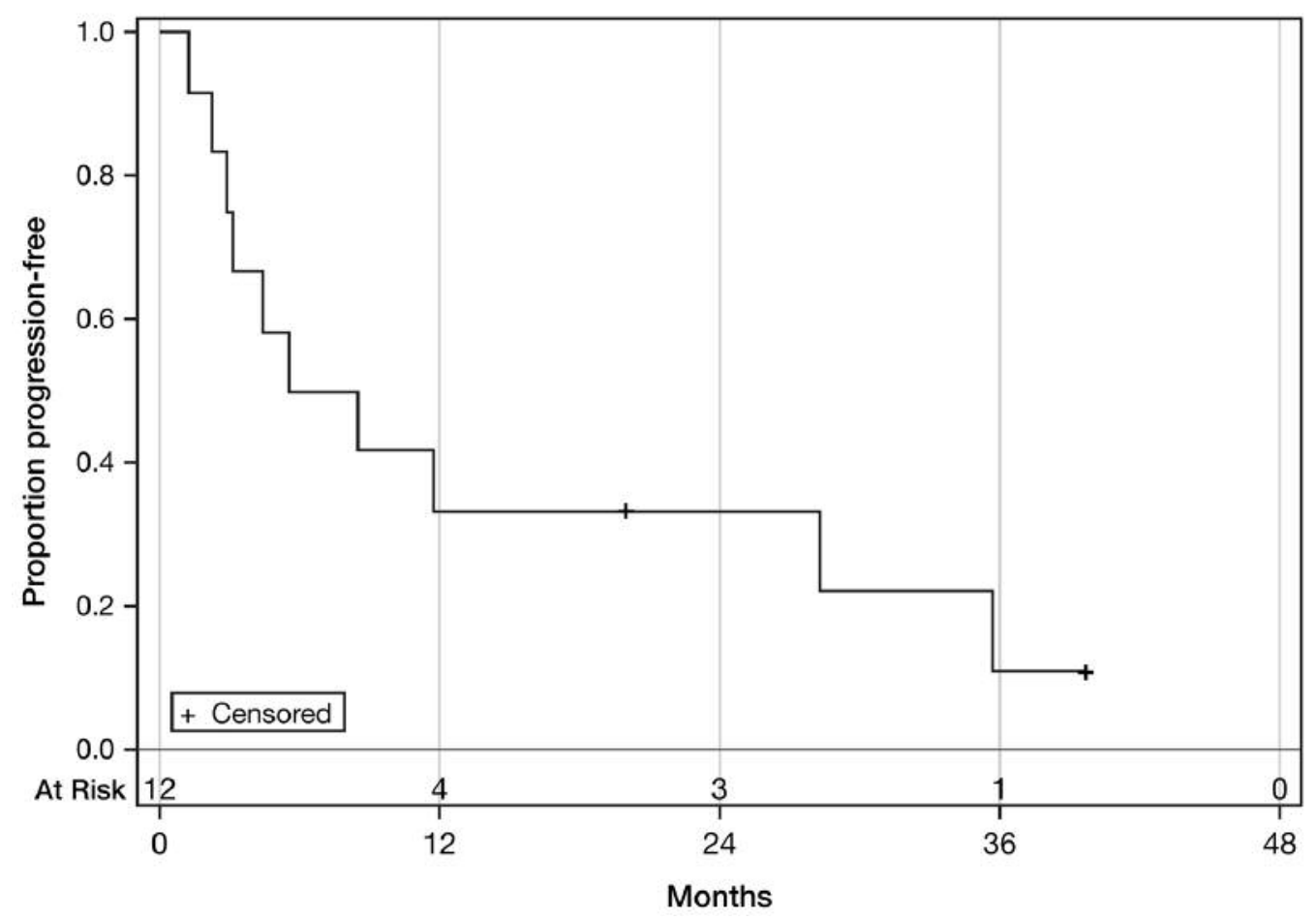

Figure 1. Progression-free survival. Median: 7 Months (95\% CI=2-28 months).

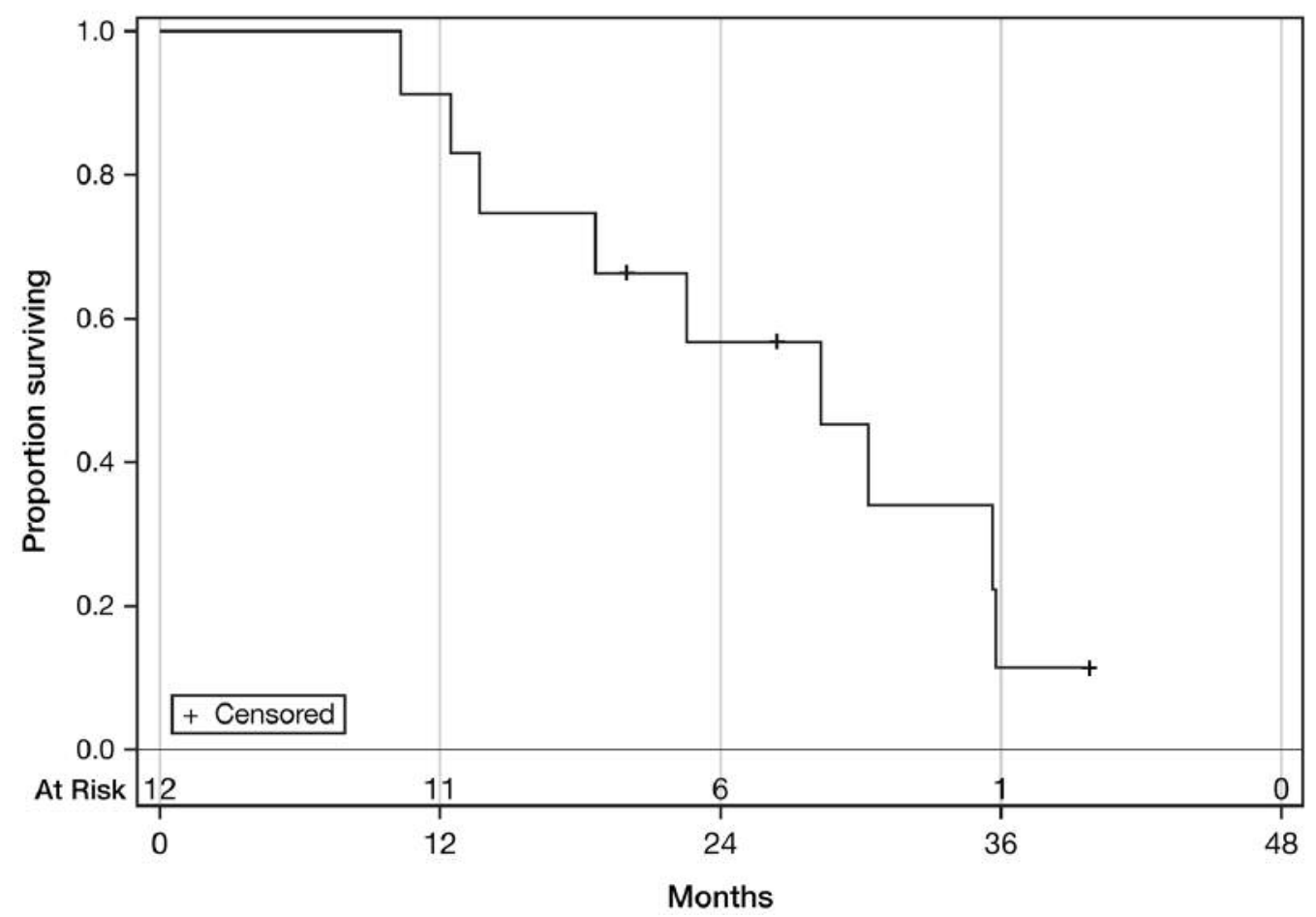

Figure 2. Overall survival. Median: 28 Months (95\% CI=12-36 months). 
Standard pre-medications were given prior to each dose administration. Patients were pre-medicated with dexamethasone, diphenhydramine, and antiemetics

Adverse events were documented per Common Terminology Criteria for Adverse Events version 4.0 (11). Supportive care in the form of anti-emetics, transfusion, or growth factor support was allowed as needed. Patients were assessed immediately prior to each chemotherapy dose for toxicity. After completing the adjuvant therapy, patients were assessed for relapse and toxicity at 1 month, then every 3 months for 1 year, and then every 6 months for 3 years.

Statistical design and analysis. This pilot study was designed to obtain preliminary data on patient tolerability of adjuvant paclitaxel with tolerability being defined as receipt of a total dose $\geq 240 \mathrm{mg} / \mathrm{m}^{2}$ which corresponds to $\geq 50 \%$ of the total planned dose. Sample size determination was based on the estimated number of patient receiving care for esophageal cancer over a 2 -year time period at our institution, i.e. 18 patients. This sample size achieves $72 \%$ power to detect a difference of $20 \%$ using a one-sided binomial test (alpha $=0.10)$ and assuming a null tolerability rate of $55 \%$.

Simple descriptive summary statistics were used to summarize patient and clinicopathological characteristics as well as adverse events. A point estimate of tolerability along with a $95 \%$ exact confidence interval (CI) was constructed. Survival probabilities were estimated and plotted using the Kaplan-Meier method. Time was calculated from treatment initiation to progression or death due to any cause for progression free survival, and to death due to any cause for overall survival. Patients without event were censored at last follow-up. Summaries and plots were derived using SAS v9.4 (SAS Institute, Cary, NC, USA).

\section{Results}

Twelve patients received at least some postoperative adjuvant paclitaxel therapy on this trial. Patient and clinical characteristics are presented in Table I. The median age of the treated patients was 57.5 years (range $=33-74$ years). Patients were predominately male $(75.0 \%)$. There was minimal improvement for these patients from pre-treatment to post-chemoradiotherapy stage.

Eleven out of the 12 patients $(92 \%, 95 \% \mathrm{CI}=62-100 \%)$ were able to complete at least $50 \%$ of the total planned dose of adjuvant chemotherapy; six patients received $480 \mathrm{mg} / \mathrm{m}^{2}$, 1 patient each received $420 \mathrm{mg} / \mathrm{m}^{2}, 360 \mathrm{mg} / \mathrm{m}^{2}, 320 \mathrm{mg} / \mathrm{m}^{2}$, $280 \mathrm{mg} / \mathrm{m}^{2}, 240 \mathrm{mg} / \mathrm{m}^{2}$, and $160 \mathrm{mg} / \mathrm{m}^{2}$. Seven patients received six treatments; chemotherapy dose was reduced in one patient, three received five treatments, one received four treatments, and one patient received two treatments but developed meningitis and was removed from the trial. Therapy was held at least once in five patients due to neutropenia.

The median progression-free survival was 7 months $(95 \%$ $\mathrm{CI}=2-28$ months). The median overall survival was 28 months (95\% CI=12-36 months). Nine patients died. Of the three patients remaining alive, one had experienced relapse by the time of writing this article. The Kaplan-Meier progression-free and overall survival curves are presented in Figures 1 and 2.
Maximum grade 3-5 paclitaxel-attributable toxicities (either a rating of possible, probable, or definite) are listed in Table II. Of the 12 patients, five experienced at least one grade 3 or 4 treatment-related toxicity. No patient experienced grade 5 toxicity. All toxicities were associated with abnormalities in laboratory values.

\section{Discussion}

Preliminary evidence suggests administration of adjuvant paclitaxel chemotherapy to patients with esophageal cancer following completion of concurrent neoadjuvant chemoradiation and esophagectomy might be well tolerated. Eleven out of the 12 patients enrolled were able to tolerate adjuvant paclitaxel, as demonstrated by receiving at least $50 \%$ of the intended total dose of adjuvant paclitaxel. This study highlights the importance of selecting appropriate patients with esophageal cancer to study adjuvant therapies following chemoradiation and esophagectomy as the tripletherapy approach (chemoradiation and esophagectomy) often leads to severe prolonged complications which might hinder conclusive adjuvant therapy trials.

The patients in this trial had all failed to achieve complete remission from their neoadjuvant therapy. They likely represent a group more resistant to chemotherapy. Therefore, it was not surprising that adjuvant single-agent paclitaxel was still associated with high rate of cancer relapse (10 out of the 12 patients had relapse) especially since 10 out of the 12 patients had already received neoadjuvant paclitaxel without achieving complete remission. This study was closed before completing accrual due to a slow accrual rate, the high rate of cancer relapse and introduction of new clinical trials testing novel agents, such as immunotherapies, in the adjuvant setting.

Trials that evaluated the benefit from adjuvant systemic treatments in esophageal cancer following neoadjuvant chemoradiation and surgery are rare (6). The trials testing chemotherapy in resectable esophageal cancer mostly focused on the neoadjuvant approach, whether as chemotherapy only or concurrent chemoradiation followed by esophagectomy (12). In the CROSS trial, the neoadjuvant treatment resulted in $29 \%$ complete response (1), which shows the critical need to use adjuvant treatments in cases of less than complete response ( $\sim 70 \%$ of the cases) to try to improve the odds of prolonging survival and cure.

In conclusion, our trial showed that screening patients with resectable esophageal cancer after triple treatment may be a successful approach to increasing the odds of completing an adjuvant esophageal cancer trial. New treatments, such as immunotherapy, might be better options for adjuvant trials, compared to cytotoxic chemotherapy, especially in patients with residual disease following neoadjuvant cytotoxic chemotherapy. 


\section{Conflict of Interest}

The Authors declare that they have no conflict of interest in regard to this study.

\section{Ethical Approval}

All procedures performed in studies involving human participants were in accordance with the ethical standards of the institutional and/or national research committee and with the 1964 Helsinki declaration and its later amendments or comparable ethical standards.

\section{Acknowledgements}

This project was supported by The Holden Comprehensive Cancer Center at The University of Iowa and its National Cancer Institute Cancer Center Support Grant (CCSG) P30CA086862.

The Authors also acknowledge the data management for this trial by Janelle Born, RN.

\section{References}

1 van Hagen P, Hulshof MC, van Lanschot JJ, Steyerberg EW, van Berge Henegouwen MI, Wijnhoven BP, Richel DJ, Nieuwenhuijzen GA, Hospers GA, Bonenkamp JJ, Cuesta MA, Blaisse RJ, Busch OR, ten Kate FJ, Creemers GJ, Punt CJ, Plukker JT, Verheul HM, Spillenaar Bilgen EJ, van Dekken H, van der Sangen MJ, Rozema T, Biermann K, Beukema JC, Piet AH, van Rij CM, Reinders JG, Tilanus HW, van der Gaast A and Group C: Preoperative chemoradiotherapy for esophageal or junctional cancer. N Engl J Med 366: 2074-2084, 2012.

2 Ancona E, Ruol A, Santi S, Merigliano S, Sileni VC, Koussis H, Zaninotto G, Bonavina L and Peracchia A: Only pathologic complete response to neoadjuvant chemotherapy improves significantly the long term survival of patients with resectable esophageal squamous cell carcinoma: final report of a randomized, controlled trial of preoperative chemotherapy versus surgery alone. Cancer 91: 2165-2174, 2001.

3 Ando $\mathrm{N}$, Iizuka $\mathrm{T}$, Ide $\mathrm{H}$, Ishida $\mathrm{K}$, Shinoda $\mathrm{M}$, Nishimaki T, Takiyama W, Watanabe H, Isono K, Aoyama N, Makuuchi H, Tanaka O, Yamana H, Ikeuchi S, Kabuto T, Nagai K, Shimada Y, Kinjo Y, Fukuda $\mathrm{H}$ and Japan Clinical Oncology Group: Surgery plus chemotherapy compared with surgery alone for localized squamous cell carcinoma of the thoracic esophagus: a Japan Clinical Oncology Group Study--JCOG9204. J Clin Oncol 21: 4592-4596, 2003.
4 Ando N, Iizuka T, Kakegawa T, Isono K, Watanabe H, Ide H, Tanaka O, Shinoda M, Takiyama W, Arimori M, Ishida K and Tsugane S: A randomized trial of surgery with and without chemotherapy for localized squamous carcinoma of the thoracic esophagus: the Japan Clinical Oncology Group Study. J Thorac Cardiovasc Surg 114: 205-209, 1997.

5 Cunningham D, Allum WH, Stenning SP, Thompson JN, Van de Velde CJ, Nicolson M, Scarffe JH, Lofts FJ, Falk SJ, Iveson TJ, Smith DB, Langley RE, Verma M, Weeden S, Chua YJ and Participants MT: Perioperative chemotherapy versus surgery alone for resectable gastroesophageal cancer. N Engl J Med 355: 11-20, 2006.

6 Horgan AM, Darling G, Wong R, Guindi M, Liu G, Jonker DJ, Lister J, Xu W, MacKay HM, Dinniwell R, Kim J, Pierre A, Shargall Y, Asmis TR, Agboola O, Seely AJ, Ringash J, Wells J, Marginean EC, Haider $M$ and Knox JJ: Adjuvant sunitinib following chemoradiotherapy and surgery for locally advanced esophageal cancer: a phase II trial. Dis Esophagus 29: 1152$1158,2016$.

7 Lee J, Lee KE, Im YH, Kang WK, Park K, Kim K and Shim YM: Adjuvant chemotherapy with 5-fluorouracil and cisplatin in lymph node-positive thoracic esophageal squamous cell carcinoma. Ann Thorac Surg 80: 1170-1175, 2005.

8 Zhang SS, Yang H, Xie X, Luo KJ, Wen J, Bella AE, Hu Y, Yang $\mathrm{F}$ and $\mathrm{Fu} \mathrm{JH}$ : Adjuvant chemotherapy versus surgery alone for esophageal squamous cell carcinoma: a meta-analysis of randomized controlled trials and nonrandomized studies. Dis Esophagus 27: 574-584, 2014.

9 Ajani J, Ilson D and Kelsen D: The activity of paclitaxel in gastrointestinal tumors. Semin Oncol 22: 46, 1995.

10 Ilson DH, Ajani J, Bhalla K, Forastiere A, Huang Y, Patel P, Martin L, Donegan J, Pazdur R, Reed C and Kelsen DP: Phase II trial of paclitaxel, fluorouracil, and cisplatin in patients with advanced carcinoma of the esophagus. J Clin Oncol 16: 1826$1834,1998$.

11 Common Terminology Criteria for Adverse Events v4.0 (CTCAE). Publication Date: May 28, 2009.

12 Gebski V, Burmeister B, Smithers BM, Foo K, Zalcberg J, Simes $\mathrm{J}$ and Australasian Gastro-Intestinal Trials Group: Survival benefits from neoadjuvant chemoradiotherapy or chemotherapy in oesophageal carcinoma: a meta-analysis. Lancet Oncol 8: 226-234, 2007
Received May 6, 2018

Revised June 16, 2018

Accepted July 23, 2018 